\title{
Resveratrol activates endogenous cardiac stem cells and improves myocardial regeneration following acute myocardial infarction
}

\author{
LIN LING ${ }^{1}$, SHAOHUA GU ${ }^{2}$ and YAN CHENG ${ }^{3}$ \\ ${ }^{1}$ Department of Cardiology, The First Affiliated Hospital of Soochow University, Suzhou, Jiangsu 215006; \\ ${ }^{2}$ Department of Nephrology, The Third People's Hospital of Kunshan; ${ }^{3}$ Department of Cardiology, \\ The Affiliated Wuxi People's Hospital of Nanjing Medical University, \\ Wuxi, Jiangsu 214000, P.R. China
}

Received October 6, 2015; Accepted October 13, 2016

DOI: $10.3892 / \mathrm{mmr} .2017 .6143$

\begin{abstract}
Stem cell antigen-1-positive $\left(\mathrm{Sca}-1^{+}\right)$cardiac stem cells (CSCs) therapy for myocardial regeneration following acute myocardial infarction (AMI) is limited by insufficient cell viability and a high rate of apoptosis, due to the poor regional microenvironment. Resveratrol, which is a compound extracted from red wine, has been reported to protect myocardial tissue post-AMI by increasing the expression of angiogenic and chemotactic factors. The present study aimed to investigate the effects of resveratrol on Sca- $1^{+} \mathrm{CSCs}$, and to optimize Sca- $1^{+}$CSCs therapy for myocardial regeneration post-AMI. C57/BL6 mice (age, 6 weeks) were divided into two groups, which received intragastric administration of PBS or $2.5 \mathrm{mg} / \mathrm{kg}$.d resveratrol. The endogenous expression of Sca- $1^{+}$CSCs in the heart was assessed on day 7. Furthermore, C57/BL6 mice underwent left anterior descending coronary artery ligation for the construction of an AMI model, and received an injection of $1 \times 10^{6} \mathrm{CSC}$ into the peri-ischemic area ( $n=8 /$ group). Mice received intragastric administration of PBS or resveratrol $(2.5 \mathrm{mg} / \mathrm{kg}$.d) for 4 weeks after cell transplantation. Echocardiography was used to evaluate cardiac function 4 weeks after cell transplantation. Capillary density and cardiomyocyte apoptosis in the peri-ischemic myocardium were assessed by cluster of differentiation 31 immunofluorescent staining and terminal deoxynucleotidyl transferase-mediated
\end{abstract}

Correspondence to: Dr Lin Ling, Department of Cardiology, The First Affiliated Hospital of Soochow University, 188 Shizi Road, Suzhou, Jiangsu 215006, P.R. China

E-mail: linglin@suda.edu.cn

Abbreviations: Sca- $^{+}$CSCs, stem cell antigen-1-positive cardiac stem cells; AMI, acute myocardial infarction; LVEDD, left ventricular end-diastolic diameter; LVESD, left ventricular end-systolic diameter; IVST, interventricular septal thickness in diastole; LVPWT, left-ventricular posterior wall thickness; FS, percent LV fractional shortening

Key words: resveratrol, Sca-1+ CSCs, myocardial infarction
dUTP nick end labeling assay, respectively. Western blot analysis was conducted to detect the protein expression levels of vascular endothelial growth factor (VEGF) and stromal cell-derived factor (SDF)-1 $\alpha$ in the myocardium. Treatment with resveratrol increased the number of endogenous Sca- $1^{+}$ CSCs in heart tissue after 7 days (PBS vs. Res, 1.85 $\pm 0.41 /$ field vs. 3.14 $\pm 0.26 /$ field, $\mathrm{P}<0.05)$. Furthermore, intragastric administration of resveratrol significantly increased left ventricle (LV) function 4 weeks after AMI, as determined by an increase in LV fractional shortening (CSCs vs. Res + CSCs, $28.82 \pm 1.58 \%$ vs. $31.18 \pm 2.02 \%, \mathrm{P}<0.05)$, reduced $\mathrm{LV}$ end-diastolic diameter (CSCs vs. Res + CSCs, $0.37 \pm 0.01 \mathrm{~mm}$ vs. $0.35 \pm 0.02 \mathrm{~mm}, \mathrm{P}<0.05)$, and reduced LV end-systolic diameter (CSCs vs. Res + CSCs, $0.26 \pm 0.01 \mathrm{~mm}$ vs. $0.23 \pm 0.02 \mathrm{~mm}$, $\mathrm{P}<0.05)$. These protective effects were predominantly achieved via an increase in capillary density (CSCs vs. Res + CSCs, $281.02 \pm 24.08 /$ field vs. $329.75 \pm 36.69 /$ field, $\mathrm{P}<0.05)$ and a reduction in cardiomyocyte apoptosis (CSCs vs. Res + CSCs, $1.5 \pm 0.54 /$ field vs. $0.83 \pm 0.40 /$ field, $\mathrm{P}<0.05)$ in peri-ischemic myocardium. Western blot analysis indicated that VEGF and SDF-1 $\alpha$ were upregulated in resveratrol-treated myocardium after a 7 day treatment or 4 weeks after AMI (7 days VEGF PBS vs. Res, $0.89 \pm 0.07$ vs. $1.21 \pm 0.02, \mathrm{P}<0.05$; SDF- $1 \alpha$ PBS vs. Res, $0.66 \pm 0.04$ vs. $1.33 \pm 0.04, \mathrm{P}<0.05$; 4 weeks VEGF CSCs vs. Res + CSCs, $0.54 \pm 0.03$ vs. $0.93 \pm 0.13, \mathrm{P}<0.05$; SDF-1 $\alpha$ CSCs vs. Res + CSCs, $0.53 \pm 0.03$ vs. $0.93 \pm 0.03, \mathrm{P}<0.05)$. Resveratrol activated endogenous CSCs, increased capillary density and decreased cardiomyocyte apoptosis in the peri-ischemic myocardium, and augmented the effects of CSCs transplantation. These effects may be caused by the upregulation of VEGF and SDF-1 $\alpha$.

\section{Introduction}

The adult heart is no longer considered a terminally differentiated organ, due to the presence of cardiac stem cells (CSCs) (1). CSCs have been considered a prospective cell source for myocardial repair following acute myocardial infarction (AMI) due to their self-renewing properties, and their multipotent ability to differentiate into cardiomyocytes and endothelial cells $(2,3)$. CSCs are positive for several stem cell antigens, including stem cell antigen (Sca)-1 and c-Kit (4). 
CSC-based therapy may be considered a novel strategy to repair infarcted myocardium post-AMI; however, this method is limited by low regenerative efficiency, due to insufficient cell viability and a high rate of apoptosis following transplantation (5).

AMI is associated with inflammation and may result in the release of high concentrations of cytokines and inflammatory factors. These molecules may act as chemotactic factors that induce the homing of several progenitor cells into the infarcted areas to participate in myocardial repair $(6,7)$. The paracrine ability of transplanted cells is considered an important factor in the improvement of cardiac function $(8,9)$. Previous studies have indicated that the predominant effect of mesenchymal stem cells in myocardial repair was associated with the release of several cytokines and chemotactic factors involved in neovascularization or chemotaxis, including vascular endothelial growth factor (VEGF) and stromal derived factor (SDF)-1 $\alpha$ (10,11). However, whether transplanted CSCs may also act in the same way and increase the release of VEGF and SDF-1 $\alpha$, resulting in the induction of microvascular sprouting, the formation of new blood vessels and an increase in progenitor cell homing remains unknown.

Resveratrol, which is a compound extracted from red wine, is considered a strong antioxidative and protective molecule in the cardiovascular system (12). A previous in vitro study demonstrated that resveratrol was able to reduce oxidative stress in several cell types, including endothelial cells, smooth muscle cells, cardiomyocytes and macrophages (13). Besides its antioxidative effects, resveratrol has been reported to exert cardioprotective effects against ischemia-reperfusion injury in animal models. Pretreatment of AMI rats with resveratrol resulted in an increase in microvessel density, and preservation of left ventricle (LV) function and blood flow $(14,15)$. In addition, resveratrol has been shown to increase the protein levels of VEGF, angiotensin II and their receptors, in order to fulfill its angiogenic effect (16). The angiogenic effect of resveratrol was achieved through increasing the bioviability and production of nitrogen monoxide, in order to modulate the VEGF signaling pathway (17). Furthermore, upregulation of VEGF may directly influence the expression of SDF-1 $\alpha$ and $\mathrm{CXC}$ chemokine receptor type 4 (CXCR-4), which are expressed on the surface of several types of stem cells, and are required for signal transduction in stem cell-associated chemotaxis, migration and homing $(18,19)$.

Therefore, the present study hypothesized that pretreatment with resveratrol may influence the angiogenic and homing ability of CSCs via the VEGF/SDF-1 $\alpha$ pathway, and may improve their transplantation efficiency following AMI. An AMI mouse model was generated and the effects of CSC regenerative therapy with resveratrol pretreatment were determined.

\section{Materials and methods}

Ethics statement. The present study was approved by the Soochow University Scientific and Animal Ethics Committee (Suzhou, China) and it was conducted in compliance with the Chinese national regulations on the use of experimental animals. Procedures for the animal studies were performed in accordance with the Guide for the Care and Use of Laboratory Animals published by the US National Institutes of Health (revised in 1996). All animals were purchased from the Laboratory Animal Center of Soochow University. They were maintained on a standard diet with access to water, under a 12-h light/dark cycle at the Animal Center of the First Affiliated Hospital of Soochow University (Suzhou, China).

CSCs culture and resveratrol administration. CSCs were isolated from hearts harvested from male C57/BL6 mice (age, 5 weeks; weight, $10-15 \mathrm{~g}$; two groups $\mathrm{n}=11$ per group) with $0.1 \%$ collagenase B (Sigma-Aldrich; Merck Millipore, Darmstadt, Germany) and $0.2 \%$ trypsin (Invitrogen; Thermo Fisher Scientific, Inc., Waltham, MA, USA) followed euthanasia with carbon dioxide. Cells were labeled with antigen-presenting cell-conjugated anti-Sca-1 (cat. no. 130092529; Miltenyi Biotec, Inc., Auburn, CA, USA) and were separated using a magnetic selection system employing anti-antigen-presenting microbeads (Miltenyi Biotec, Inc.). Selected cells were cultured in Dulbecco's modified Eagle's medium/F12 supplemented with $10 \%$ fetal bovine serum (Gibco; Thermo Fisher Scientific, Inc.), $10 \mathrm{ng} / \mathrm{ml}$ basic fibroblast growth factor, $10 \mathrm{ng} / \mathrm{ml}$ leukemia inhibitory factor, $10 \mathrm{ng} / \mathrm{ml}$ cardiotroponin, $10 \mathrm{ng} / \mathrm{ml}$ epidermal growth factor (PeproTech, Rocky Hill, NJ, USA) and a $1 \%$ antibiotic solution of penicillin and streptomycin (Hyclone; GE Healthcare, Logan, UT, USA). The C57/BL6 mice (age, 5 weeks, two groups; $n=8$ per group) were treated with $2.5 \mathrm{mg} / \mathrm{kg}$.d resveratrol (Baile Company, China) by intragastric administration with a stomach needle. The drug was dissolved in $0.5 \mathrm{ml}$ PBS. The control group received the same volume of PBS. All mice (age, 5 weeks) received intragastric administration of resveratrol or PBS. Some $(n=3$ per group) were sacrificed in order to quantify CSCs after 7 days. Other mice ( $n=8$ per group) underwent AMI construction and cell transplantation on day 7 and continued to be treated with administration of resveratrol or PBS for 4 weeks.

Measurement of CSCs after resveratrol pretreatment. A total of 7 days after resveratrol administration, mice were sacrificed with $\mathrm{CO}_{2}$ frozen cardiac tissue specimens ( $\mathrm{n}=3$ /group) were obtained and sliced into $2 \mu \mathrm{m}$ sections to quantify the number of Sca- $1^{+}$ CSCs in the myocardium using an anti-Sca-1 immunofluorescent antibody (1:50; eBioscience, Inc., San Diego, CA, USA; cat. no. 11-5981-81). Positive cells were stained red and were counted in 10 random 100x fields using a fluorescence microscope. The average number was obtained for statistical analysis.

AMI construction and cell transplantation. An AMI model was constructed by surgically ligating the left anterior descending coronary artery (LAD) with a prolene suture. Successful ligation was verified by observation of a color change from red to white in the infarct area. C57/BL6 mice (age, 6 weeks; weight, 15-20 g) were randomly assigned into two groups ( $\mathrm{n}=8 /$ group), which received either PBS or $2.5 \mathrm{mg} / \mathrm{kg}$.d resveratrol via intragastric administration for 7 days prior to and 4 weeks after LAD ligation. All mice received an injection with $1 \times 10^{6} \mathrm{CSC}$ into the peri-ischemic area and were sacrificed with $\mathrm{CO}_{2} 4$ weeks after cell transplantation for histological analysis.

Assessment of cardiac function with echocardiography. A total of 4 weeks after LAD ligation, transthoracic echocardiography 
Table I. Echocardiography parameters following acute myocardial infarction.

\begin{tabular}{lccccr}
\hline Group & $\%$ FS & LVESD $(\mathrm{mm})$ & LVEDD $(\mathrm{mm})$ & IVST $(\mathrm{mm})$ & LVPWT (mm) \\
\hline CSCs (baseline) & $27.48 \pm 1.05$ & $0.21 \pm 0.02$ & $0.31 \pm 0.01$ & $0.07 \pm 0.006$ & $0.09 \pm 0.004$ \\
Res + CSCs (baseline) & $28.64 \pm 0.83$ & $0.21 \pm 0.02$ & $0.32 \pm 0.03$ & $0.07 \pm 0.004$ & $0.09 \pm 0.005$ \\
CSCs (4w) & $28.82 \pm 1.58$ & $0.26 \pm 0.01$ & $0.37 \pm 0.01$ & $0.07 \pm 0.009$ & $0.09 \pm 0.005$ \\
Res + CSCs (4w) & $31.18 \pm 2.02^{\mathrm{a}}$ & $0.23 \pm 0.02^{\mathrm{a}}$ & $0.35 \pm 0.02^{\mathrm{a}}$ & $0.07 \pm 0.008$ & $0.09 \pm 0.005$
\end{tabular}

${ }^{\mathrm{a}} \mathrm{P}<0.05$, vs. CSCs (4w). CSCs, cardiac stem cells; Res, resveratrol; w, weeks; FS, fractional shortening; LVESD, left ventricular end-systolic diameter; LVEDD, left ventricular end-diastolic diameter; IVST, interventricular septal thickness in diastole; LVPWT, LV posterior wall thickness.

(SONOS 5500, Philips Medical Systems International BV, Eindhoven, The Netherlands) was used to evaluate the cardiac function of experimental mice by an observer blinded to the experiment. LV end-diastolic diameter (LVEDD), LV end-systolic diameter (LVESD), interventricular septal thickness in diastole (IVST), LV posterior wall thickness (LVPWT) and the percentage of LV fractional shortening (FS) were detected. All measurements were repeated for at least three consecutive pulsation cycles and the data were averaged for statistical analysis.

Capillary density assessment. Cluster of differentiation (CD)31 immunofluorescent staining was conducted to determine capillary density. Frozen tissue specimens of the infarcted area were sliced into $2 \mu \mathrm{m}$ sections and were labeled with rat anti-mouse CD31 (1:800; Abcam, Cambridge, MA, USA; cat. no. ab8365) and Alexa Fluor 594 anti-rat immunoglobulin G (Invitrogen; Thermo Fisher Scientific, Inc.; cat. no. SA000064). CD31-positive cells were stained red and the number of capillaries was counted using Image Pro Plus Software version 6.0 (Media Cybernetics, Inc., Rockville, MD, USA) in 10 random 100x fields using a fluorescence microscope and averaged for statistical analysis.

Terminal deoxynucleotidyl transferase-mediated dUTP nick end labeling (TUNEL) assay. The present study detected cardiomyocyte apoptosis in the peri-ischemic myocardium using the TUNEL assay (Promega Corporation, Madison, WI, USA), according to the manufacturer's protocol. Nuclei of apoptotic cardiomyocytes were stained dark brown. The number of TUNEL-positive cardiomyocyte nuclei per 200x field was counted and used for statistical analysis.

Western blot analysis. Cardiac tissues were lysed using lysis buffer with phosphatase inhibitor (Roche Diagnostics, Basel, Switzerland). A bicinchoninic acid protein assay kit (Pierce; Thermo Fisher Scientific, Inc.) was used to determine protein concentrations. Proteins (30 $\mu \mathrm{g}$ samples) were separated by $10 \%$ SDS-PAGE and were transferred to a polyvinylidene fluoride membrane (EMD Millipore, Billerica, MA, USA). The membrane was blocked with $5 \%$ nonfat dried milk dissolved in Tris-buffered saline containing $0.1 \%$ Tween-20 at room temperature for $2 \mathrm{~h}$. Subsequently, the membrane was incubated with primary antibodies against VEGF $(1: 2,000$; Abcam; cat. no. ab2350) and SDF-1 $\alpha$ (1:1,500; Abcam; cat.

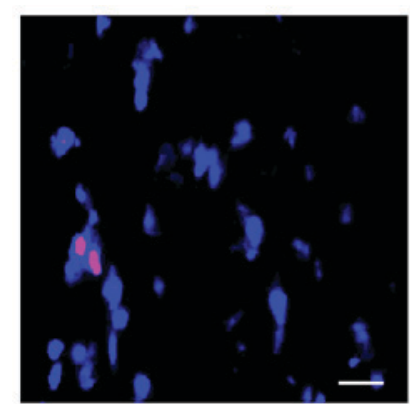

PBS

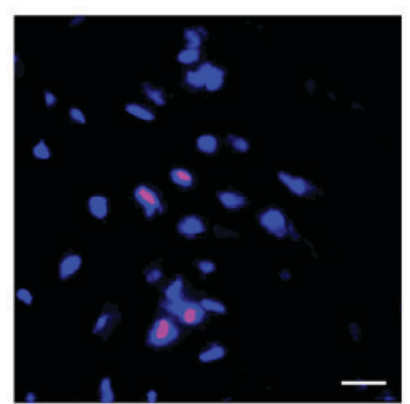

Resveratrol

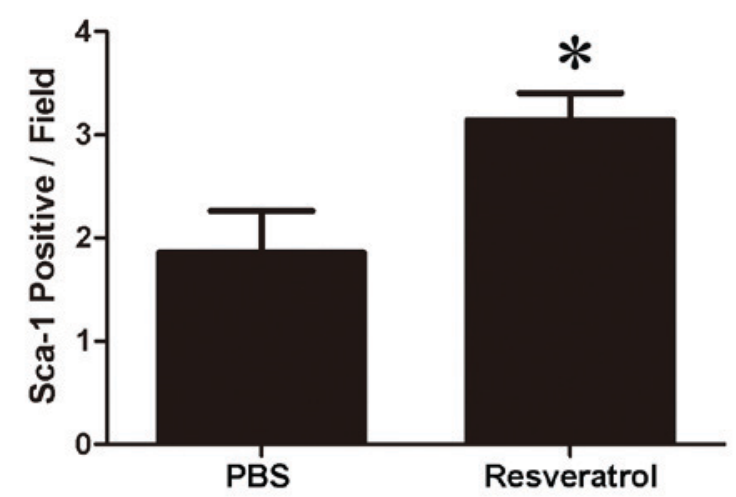

Figure 1. Resveratrol treatment for 7 days increased the number of Sca- $1^{+}$ cardiac stem cells in the myocardium. ${ }^{*} \mathrm{P}<0.05$ vs. the PBS group. Scale bar, $100 \mu \mathrm{m}$ Sca-1, stem cell antigen-1.

no. ab9797) overnight at $4^{\circ} \mathrm{C}$, followed by an incubation with peroxidase-conjugated secondary antibodies for $2 \mathrm{~h}$ at room temperature. Bands were detected using a chemiluminescent western blot detection system (Pierce; Thermo Fisher Scientific, Inc.). Protein expression was normalized to GAPDH. Assays were repeated three times and band intensities were quantified using the Photo-Image system (Siemens AG, Munich, Germany).

Statistical analysis. Data are presented as the mean \pm standard deviation and were analyzed using SPSS 17.0 statistical software (SPSS, Inc., Chicago, IL, USA). Comparisons between two groups were performed using an unpaired Student's t-test. $\mathrm{P}<0.05$ was considered to indicate a statistically significant difference. 
A

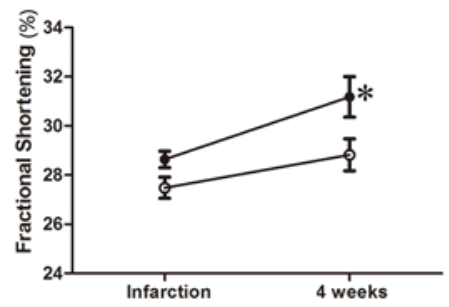

D

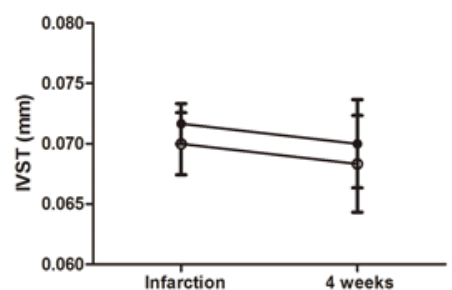

B

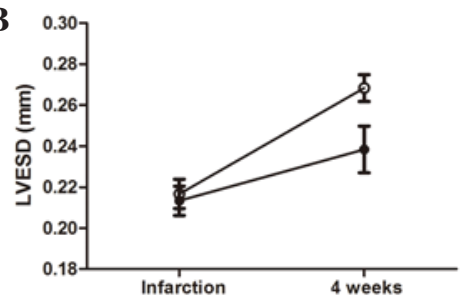

$\mathbf{E}$

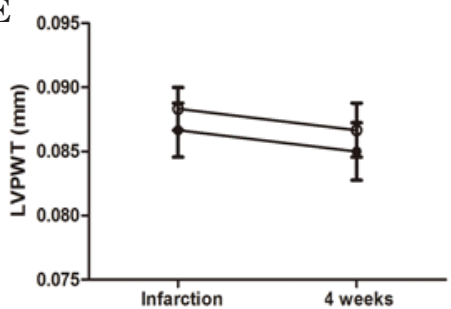

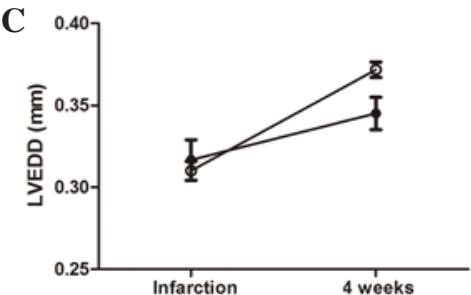

- $\operatorname{cscs}$

$\rightarrow$ Resveratrol+CSCs

Figure 2. Stem cell antigen $-1^{+}$CSCs transplantation combined with resveratrol treatment improved cardiac performance 4 weeks after acute myocardial infarction, as determined by measuring (A) fractional shortening (B) LVESD (C) and LVEDD ("P $<0.05$ vs. the CSCs group). There was no significant difference in (D) IVST and (E) LVPWT between the groups. CSCs, cardiac stem cells; LVESD, left ventricular end-systolic diameter; LVEDD, left ventricular end-diastolic diameter; IVST, interventricular septal thickness in diastole; LVPWT, LV posterior wall thickness.

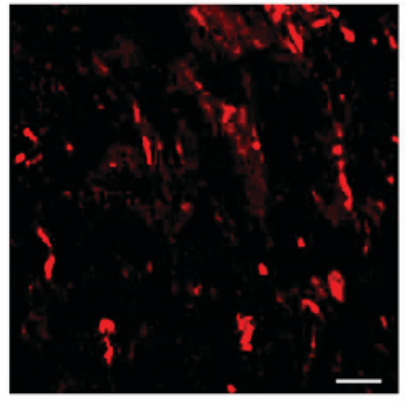

cscs

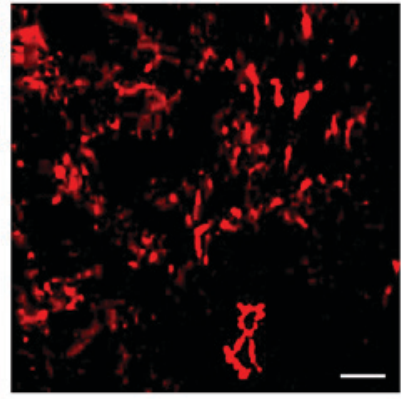

Resveratrol+CSCs

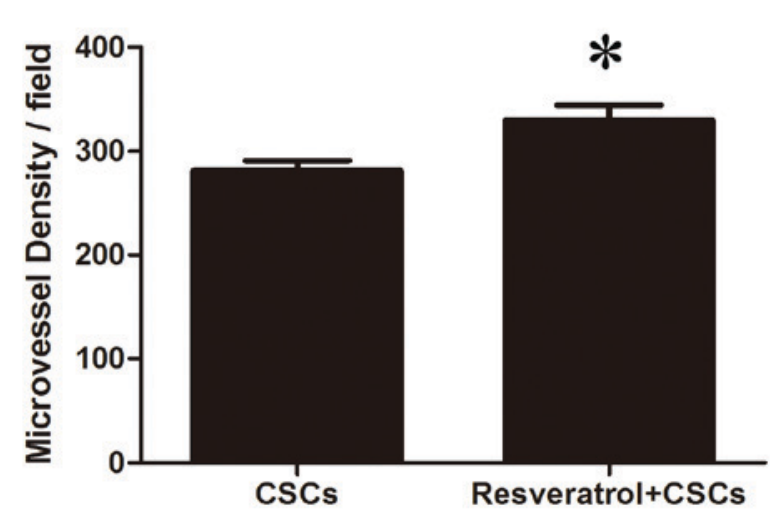

Figure 3. Stem cell antigen $-1^{+}$CSCs transplantation combined with resveratrol treatment increased capillary density in infarcted myocardium 4 weeks after acute myocardial infarction. ${ }^{*} \mathrm{P}<0.05$ vs. the CSCs group. Scale bar, $100 \mu \mathrm{m}$. CSCs, cardiac stem cells.

\section{Results}

Pretreatment with resveratrol increases the number of Sca- $1^{+}$ CSCs in myocardium. The present study examined the number of Sca- $1^{+}$CSCs following resveratrol pretreatment. Sca- $1^{+}$

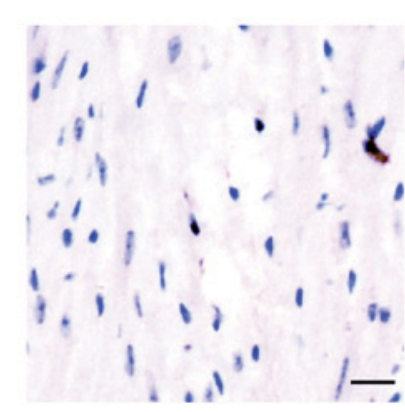

CSCs

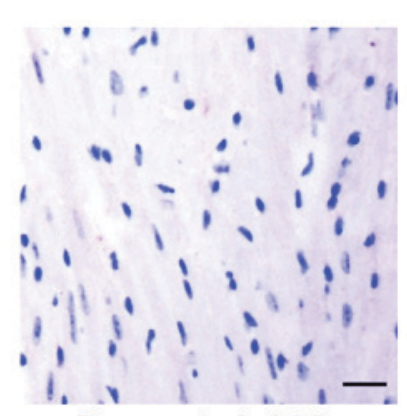

Resveratrol+CSCs

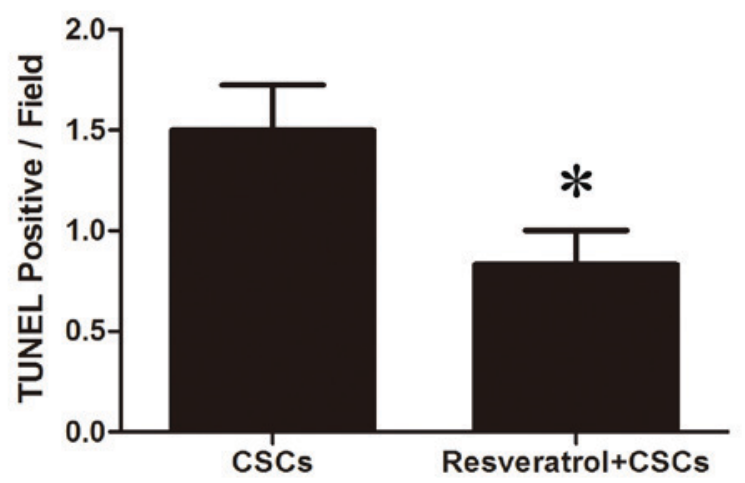

Figure 4. Stem cell antigen- $1^{+}$CSCs transplantation combined with resveratrol treatment decreased cardiomyocyte apoptosis in infarcted myocardium 4 weeks after acute myocardial infarction. ${ }^{*} \mathrm{P}<0.05$ vs. the CSCs group. Scale bar, $100 \mu \mathrm{m}$. CSCs, cardiac stem cells; Tunel, terminal deoxynucleotidyl transferase-mediated dUTP nick end labeling.

CSCs were stained red and the nuclei were stained blue. Pretreatment with resveratrol for 7 consecutive days resulted in an increase in the number of Sca- $1^{+}$CSCs in the myocardium (PBS vs. resveratrol, 1.85 $\pm 0.41 /$ field vs. 3.14 $\pm 0.26 /$ field, $\mathrm{P}<0.05$, Fig. 1). 
A
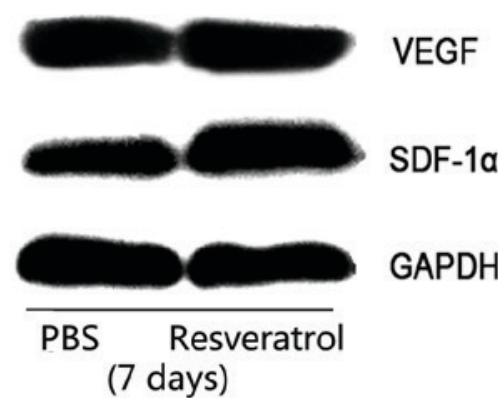

C

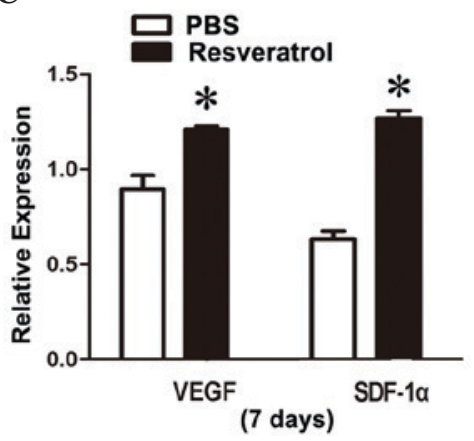

B

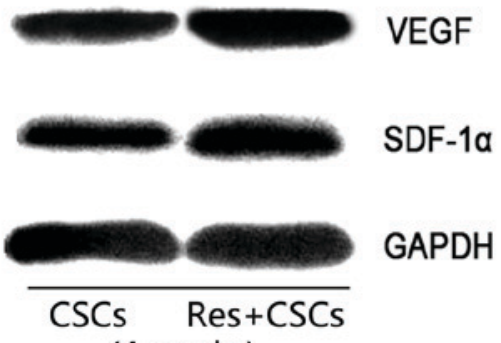

(4 weeks)

D

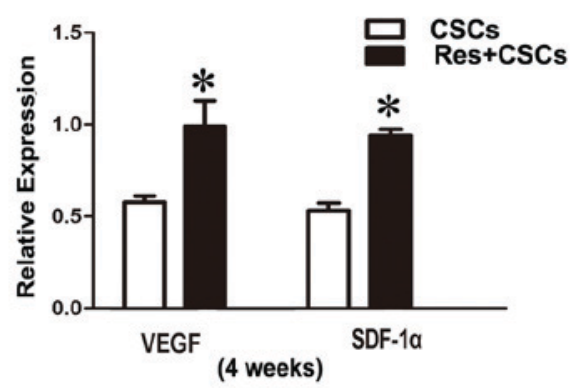

Figure 5. VEGF and SDF-1 $\alpha$ protein expression was upregulated in resveratrol-treated myocardium after (A) 7 days treatment, and (B) 4 weeks after acute myocardial infarction. Quantification of western blotting for after (C) 7 days treatment and (D) 4 weeks. * $\mathrm{P}<0.05$. Res, resveratrol; CSCs, cardiac stem cells; VEGF, vascular endothelial growth factor; SDF-1 $\alpha$, stromal cell-derived factor-1 $\alpha$.

CSCs combined with resveratrol protect cardiac function post-AMI. A total of 4 weeks after LAD ligation, cardiac function of the mice was evaluated by echocardiography; the results are presented in Fig. 2. CSCs transplantation combined with resveratrol administration exerted a protective effect on mice, as compared with CSCs transplantation only. Resveratrol and CSCs transplantation preserved LV FS, and reduced LVEDD and LVESD (CSCs vs. resveratrol + CSCs, P<0.05, Fig. 2). The detailed data are presented in Table I.

CSCs combined with resveratrol increase capillary density in infarcted myocardium. CD31 immunofluorescent staining was used to measure capillary density in the infarcted myocardium. As presented in Fig. 3, capillaries were stained red. A total of 4 weeks after LAD ligation, an increased number of CD31-positive cells was detected in the resveratrol-treated myocardium (CSCs vs. resveratrol + CSCs, 281.02 $\pm 24.08 /$ field vs. $329.75 \pm 36.69 /$ field, $\mathrm{P}<0.05)$.

CSCs combined with resveratrol reduce cardiomyocyte apoptosis. Cardiomyocyte apoptosis was measured by TUNEL assay. TUNEL-positive cells were stained brown. As presented in Fig. 4, the number of TUNEL-positive cells in the peri-ischemic area was reduced in the resveratrol + CSCs group compared with in the CSCs group (CSCs vs. resveratrol + CSCs, $1.5 \pm 0.54 /$ field vs. $0.83 \pm 0.40 /$ field, $\mathrm{P}<0.05$ ).

VEGF and SDF-1 a expression is upregulated in resveratrol-treated myocardium. Western blotting was used to evaluate the protein expression levels of VEGF and SDF-1 $\alpha$ in myocardium 7 days after resveratrol administration, and 4 weeks after AMI. Western blot analysis indicated that VEGF and SDF-1 $\alpha$ were upregulated in resveratrol-treated myocardium after 7 days of treatment, and 4 weeks after AMI (7 days
VEGF PBS vs. resveratrol, $0.89 \pm 0.07$ vs. $1.21 \pm 0.02, \mathrm{P}<0.05$; SDF-1 $\alpha$ PBS vs. resveratrol, $0.66 \pm 0.04$ vs. $1.33 \pm 0.04, \mathrm{P}<0.05$; 4 weeks VEGF CSCs vs. resveratrol + CSCs, $0.54 \pm 0.03$ vs. $0.93 \pm 0.13, \mathrm{P}<0.05$; SDF- $1 \alpha \mathrm{CSCs}$ vs. resveratrol $+\mathrm{CSCs}$, $0.53 \pm 0.03$ vs. $0.93 \pm 0.03, \mathrm{P}<0.05$, Fig. 5 ).

\section{Discussion}

Since the adult heart was recognized as containing its own stem cells (20), CSCs therapy for AMI has been considered as a novel treatment strategy. CSCs are able to regenerate several types of cell, including cardiomyocytes, endothelial cells and smooth muscle cells, and directly participate in myocardial and endothelial repair $(21,22)$. Cell therapy of AMI with CSCs has been reported to ameliorate cardiac function and tissue remodeling (23). However, just like other stem cell-based therapies for AMI, CSCs-based therapy is hindered by limited transplantation efficiency, and researchers have aimed to solve this problem with appropriate cell modification (24). Resveratrol is a compound that exerts numerous effects, including anti-inflammatory, antitumor and immunomodulatory activities. Resveratrol has also been reported to exert strong cardioprotective effects in the cardiovascular system $(25,26)$. The present study combined this cardioprotective agent with CSCs-based therapy. A mouse model of AMI treated with CSCs transplantation plus resveratrol exhibited preserved cardiac function, increased capillary density and decreased cardiomyocyte apoptosis in the peri-ischemic myocardium. In addition, VEGF and SDF-1 $\alpha$ expression was upregulated. These results suggested that this combination therapy may augment the effects of CSCs-mediated myocardial regeneration, and may fulfill the potential of CSCs cell therapy for AMI.

VEGF is widely known as an angiogenic molecule that serves an important role in neovascularization and angiogenesis 
in several physiological and pathological conditions (27). AMI is a process associated with severe ischemia and hypoxia, which induces upregulation of the transcription factor hypoxia-inducible factor-1 (HIF) (28). HIF is an upstream signaling molecule of VEGF, which can modulate the synthesis and release of VEGF and downstream signaling pathways, such as endothelial nitric oxide synthase (29). Upregulation of VEGF directly leads to increased angiogenesis, as well as capillary density, in the ischemic myocardium. Resveratrol has been revealed to elevate the protein expression levels of VEGF, thus modulating the interaction between VEGF and VEGF receptors in an AMI model, which may lead to improved vessel collateralization and angiogenesis in infarcted myocardium $(30,31)$. The present study also demonstrated that CSCs transplantation plus resveratrol pretreatment upregulated the synthesis and release of VEGF. This increase in VEGF may subsequently lead to increased capillary density after cell transplantation, which is evidence of further angiogenesis.

Besides VEGF, SDF-1 $\alpha$ has been reported to modulate the homing of stem cells to the site of injury by chemokinesis and chemotaxis, through uniquely binding to the specific cell surface receptor CXCR-4 $(32,33)$. The SDF-1 $\alpha / C X C R-4$ targeting system is vital in cardiac development, as confirmed by the fetal lethality of SDF-1 $\alpha$ or CXCR- 4 knockout in mice (34). SDF-1 $\alpha$ is able to direct several types of progenitor or stem cell to the targeted area with accurate chemotaxis, and maintain these stem cells in the regional ischemic area (35). It has previously been reported that gene expression of SDF-1 $\alpha$ is significantly increased in infarcted myocardium following AMI (36). Overexpression of the SDF-1 $\alpha$ gene in damaged heart tissue may induce hematopoietic stem cell homing to local ischemic myocardium. Similar to VEGF, SDF-1 $\alpha$ gene expression is also regulated by HIF (37). Regional overexpression of HIF in the ischemic and hypoxic myocardium has been shown to result in selective expression of SDF-1 $\alpha$ in the ischemic myocardium, and the level of SDF-1 $\alpha$ was in direct proportion to local reduced oxygen tension (38). This overexpression of SDF-1 $\alpha$ increases the targeting, migration and homing of circulating and resident progenitor cells to gather at the local ischemic myocardium and participate in tissue recovery. In addition, insufficient release of SDF-1 $\alpha$ has been reported to decrease the mobilization of progenitor cells (39). SDF-1 $\alpha$ has been confirmed to induce migration of Sca- $1^{+}$ CSCs in vitro. Upregulation of VEGF may also elevate the expression of CXCR-4 and SDF-1 $\alpha$ to further enhance their effects on stem cell homing, migration and chemotaxis (40).

In conclusion, the present study demonstrated that CSCs therapy combined with resveratrol may augment the effects of CSCs transplantation through increasing the synthesis of VEGF and SDF-1 $\alpha$. CSCs transplantation combined with resveratrol pretreatment improved cardiac performance in a mouse model of AMI, as compared with CSCs therapy alone. The results suggested that resveratrol-modified CSCs therapy may further augment the efficiency of CSCs therapy for AMI, and may provide an improved strategy for cell-based treatment of AMI.

\section{Acknowledgements}

The present study was supported by the Youth Science and Technology of Suzhou Science and Education Project (grant no. KJXW2013004), the Youth Science Foundation of Jiangsu Province, China (grant no. BK20140296) and the Science Foundation for Youth Teacher of Soochow University (grant no. SDY2013A29).

\section{References}

1. Waring CD, Vicinanza C, Papalamprou A, Smith AJ, Purushothaman S, Goldspink DF, Nadal-Ginard B, Torella D and Ellison GM: The adult heart responds to increased workload with physiologic hypertrophy, cardiac stem cell activation, and new myocyte formation. Eur Heart J 35: 2722-2731, 2014.

2. Noseda M, Abreu-Paiva M and Schneider MD: The quest for the adult cardiac stem cell. Circ J 79: 1422-1430, 2015.

3. Nadal-Ginard B, Ellison GM and Torella D: The cardiac stem cell compartment is indispensable for myocardial cell homeostasis, repair and regeneration in the adult. Stem Cell Res 13: 615-630, 2014.

4. Hattori F: CD117, adult cardiac stem cell marker, is transiently expressed in methothelial epicardial cells. J Mol Cell Cardiol 49: 711-712, 2010.

5. Müller-Ehmsen J, Krausgrill B, Burst V, Schenk K, Neisen UC, Fries JW, Fleischmann BK, Hescheler J and Schwinger RH: Effective engraftment but poor mid-term persistence of mononuclear and mesenchymal bone marrow cells in acute and chronic rat myocardial infarction. J Mol Cell Cardiol 41: 876-884, 2006.

6. Duran JM, Makarewich CA, Sharp TE, Starosta T, Zhu F, Hoffman NE, Chiba Y, Madesh M, Berretta RM, Kubo H and Houser SR: Bone-derived stem cells repair the heart after myocardial infarction through transdifferentiation and paracrine signaling mechanisms. Circ Res 113: 539-552, 2013.

7. Doyle B,Sorajja P,Hynes B,Kumar AH,AraozPA,StalboergerPG, Miller D, Reed C, Schmeckpeper J, Wang S, et al: Progenitor cell therapy in a porcine acute myocardial infarction model induces cardiac hypertrophy, mediated by paracrine secretion of cardiotrophic factors including TGFbeta1. Stem Cells Dev 17: 941-951, 2008.

8. Gnecchi M, Zhang Z, Ni A and Dzau VJ: Paracrine mechanisms in adult stem cell signaling and therapy. Circ Res 103: 1204-1219, 2008.

9. Mazo M, Cemborain A, Gavira JJ, Abizanda G, Araña M, Casado M, Soriano M, Hernández S, Moreno C, Ecay M, et al: Adipose stromal vascular fraction improves cardiac function in chronic myocardial infarction through differentiation and paracrine activity. Cell Transplant 21: 1023-1037, 2012.

10. Boyle AJ, Yeghiazarians Y, Shih H, Hwang J, Ye J, Sievers R, Zheng D, Palasubramaniam J, Palasubramaniam D, Karschimkus C, et al: Myocardial production and release of MCP-1 and SDF-1 following myocardial infarction: Differences between mice and man. J Transl Med 9: 150, 2011.

11. Takahashi M: Role of the SDF-1/CXCR4 system in myocardial infarction. Circ J 74: 418-423, 2010.

12. Escoté X, Miranda M, Menoyo S, Rodríguez-Porrata B, Carmona-Gutiérrez D, Jungwirth H, Madeo F, Cordero RR, Mas A, Tinahones F, et al: Resveratrol induces antioxidant defence via transcription factor Yap1p. Yeast 29: 251-263, 2012.

13. Wang Z, Chen Y, Labinskyy N, Hsieh TC, Ungvari Z and Wu JM: Regulation of proliferation and gene expression in cultured human aortic smooth muscle cells by resveratrol and standardized grape extracts. Biochem Biophys Res Commun 346: 367-376, 2006.

14. Bradamante S, Barenghi L and Villa A: Cardiovascular protective effects of resveratrol. Cardiovasc Drug Rev 22: 169-188, 2004.

15. Das S and Das DK: Resveratrol: A therapeutic promise for cardiovascular diseases. Recent Pat Cardiovasc Drug Discov 2: 133-138, 2007.

16. Fukuda S, Kaga S, Zhan L, Bagchi D, Das DK, Bertelli A and Maulik N: Resveratrol ameliorates myocardial damage by inducing vascular endothelial growth factor-angiogenesis and tyrosine kinase receptor Flk-1. Cell Biochem Biophys 44: 43-49, 2006.

17. Das S, Alagappan VK, Bagchi D, Sharma HS, Maulik N and Das DK: Coordinated induction of iNOS-VEGF-KDR-eNOS after resveratrol consumption: A potential mechanism for resveratrol preconditioning of the heart. Vascul Pharmacol 42: 281-289, 2005. 
18. Brunner S, Winogradow J, Huber BC, Zaruba MM, Fischer R, David R, Assmann G, Herbach N, Wanke R, Mueller-Hoecker J and Franz WM: Erythropoietin administration after myocardial infarction in mice attenuates ischemic cardiomyopathy associated with enhanced homing of bone marrow-derived progenitor cells via the CXCR-4/SDF-1 axis. FASEB J 23: 351-361, 2009.

19. Shen L, Gao Y, Qian J, Sun A and Ge J: A novel mechanism for endothelial progenitor cells homing: The SDF-1/CXCR4-Rac pathway may regulate endothelial progenitor cells homing through cellular polarization. Med Hypotheses 76: 256-258, 2011.

20. Lyngbaek S, Schneider M, Hansen JL and Sheikh SP: Cardiac regeneration by resident stem and progenitor cells in the adult heart. Basic Res Cardiol 102: 101-114, 2007.

21. Torella D, Ellison GM, Karakikes I and Nadal-Ginard B Resident cardiac stem cells. Cell Mol Life Sci 64: 661-673, 2007.

22. Torella D, Ellison GM, Méndez-Ferrer S, Ibanez B and Nadal-Ginard B: Resident human cardiac stem cells: Role in cardiac cellular homeostasis and potential for myocardial regeneration. Nat Clin Pract Cardiovasc Med 3 (Suppl 1): S8-S13, 2006.

23. Chamuleau SA, Vrijsen KR, Rokosh DG, Tang XL, Piek JJ and Bolli R: Cell therapy for ischaemic heart disease: Focus on the role of resident cardiac stem cells. Neth Heart J 17: 199-207, 2009.

24. Tang JM, Wang JN, Zhang L, Zheng F, Yang JY, Kong X, Guo LY, Chen L, Huang YZ, Wan Y and Chen SY: VEGF/SDF-1 promotes cardiac stem cell mobilization and myocardial repair in the infarcted heart. Cardiovasc Res 91: 402-411, 2011

25. Mokni M, Limam F, Elkahoui S, Amri M and Aouani E: Strong cardioprotective effect of resveratrol, a red wine polyphenol, on isolated rat hearts after ischemia/reperfusion injury. Arch Biochem Biophys 457: 1-6, 2007.

26. Wu JM and Hsieh TC: Resveratrol: A cardioprotective substance. Ann N Y Acad Sci 1215: 16-21, 2011.

27. Zhao T, Zhao W, Chen Y, Ahokas RA and Sun Y: Vascular endothelial growth factor (VEGF)-A: Role on cardiac angiogenesis following myocardial infarction. Microvasc Res 80: 188-194, 2010.

28. Lakkisto P, Kytö V, Forsten H, Siren JM, Segersvärd H, Voipio-Pulkki LM, Laine M, Pulkki K and Tikkanen I: Heme oxygenase-1 and carbon monoxide promote neovascularization after myocardial infarction by modulating the expression of HIF-1alpha, SDF-1alpha and VEGF-B. Eur J Pharmacol 635 156-164, 2010.

29. Knudsen AR, Kannerup AS, Grønbaek H, Andersen KJ, Funch-Jensen P, Frystyk J, Flyvbjerg A and Mortensen FV: Effects of ischemic pre- and postconditioning on HIF-1 $\alpha$, VEGF and TGF- $\beta$ expression after warm ischemia and reperfusion in the rat liver. Comp Hepatol 10: 3, 2011.
30. Kaga S, Zhan L, Matsumoto $M$ and Maulik N: Resveratrol enhances neovascularization in the infarcted rat myocardium through the induction of thioredoxin-1, heme oxygenase-1 and vascular endothelial growth factor. J Mol Cell Cardiol 39: 813-822, 2005.

31. Penumathsa SV, Thirunavukkarasu M, Koneru S, Juhasz B, Zhan L, Pant R, Menon VP, Otani H and Maulik N: Statin and resveratrol in combination induces cardioprotection against myocardial infarction in hypercholesterolemic rat. J Mol Cell Cardiol 42: 508-516, 2007.

32. Cottler-Fox MH, Lapidot T, Petit I, Kollet O, DiPersio JF, Link D and Devine S: Stem cell mobilization. Hematology Am Soc Hematol Educ Program: 419-437, 2003

33. Zou YR, Kottmann AH, Kuroda M, Taniuchi I and Littman DR: Function of the chemokine receptor CXCR4 in haematopoiesis and in cerebellar development. Nature 393: 595-599, 1998.

34. Lapidot T: Mechanism of human stem cell migration and repopulation of NOD/SCID and B2mnull NOD/SCID mice. The role of SDF-1/CXCR4 interactions. Ann N Y Acad Sci 938: 83-95, 2001

35. Askari AT, Unzek S, Popovic ZB, Goldman CK, Forudi F, Kiedrowski M, Rovner A, Ellis SG, Thomas JD, DiCorleto PE, et al: Effect of stromal-cell-derived factor 1 on stem-cell homing and tissue regeneration in ischaemic cardiomyopathy. Lancet 362: 697-703, 2003.

36. Pillarisetti $\mathrm{K}$ and Gupta SK: Cloning and relative expression analysis of rat stromal cell derived factor-1 (SDF-1)1: SDF-1 alpha mRNA is selectively induced in rat model of myocardial infarction. Inflammation 25: 293-300, 2001.

37. Loh SA, Chang EI, Galvez MG, Thangarajah H, El-ftesi S, Vial IN, Lin DA and Gurtner GC: SDF-1 alpha expression during wound healing in the aged is HIF dependent. Plast Reconstr Surg 123 (Suppl 2): S65-S75, 2009.

38. Ceradini DJ, Kulkarni AR, Callaghan MJ, Tepper OM, Bastidas N, Kleinman ME, Capla JM, Galiano RD, Levine JP and Gurtner GC: Progenitor cell trafficking is regulated by hypoxic gradients through HIF-1 induction of SDF-1. Nat Med 10: 858-864, 2004.

39. Hattori K, Heissig B, Tashiro K, Honjo T, Tateno M, Shieh JH, Hackett NR, Quitoriano MS, Crystal RG, Rafii S and Moore MA: Plasma elevation of stromal cell-derived factor-1 induces mobilization of mature and immature hematopoietic progenitor and stem cells. Blood 97: 3354-3360, 2001.

40. Tang J, Wang J, Kong X, Yang J, Guo L, Zheng F, Zhang L, Huang Y and Wan Y: Vascular endothelial growth factor promotes cardiac stem cell migration via the PI3K/Akt pathway. Exp Cell Res 315: 3521-3531, 2009. 